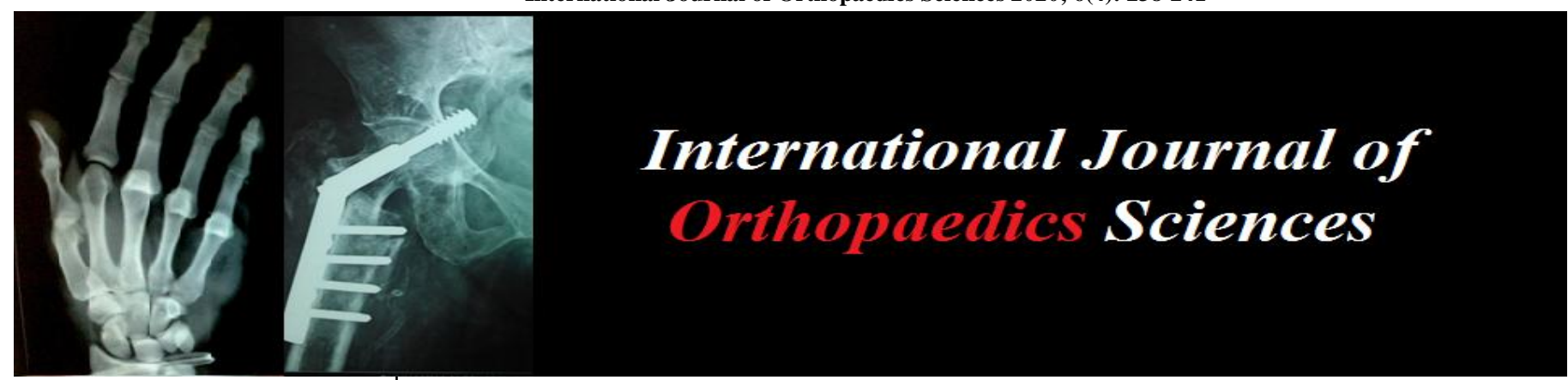

E-ISSN: 2395-1958

P-ISSN: 2706-6630

IJOS 2020; 6(4): 238-241

(C) 2020 IJOS

www.orthopaper.com

Received: 25-07-2020

Accepted: 29-08-2020

Shreesha Tokur

Assistant Professor, Karwar

Institute of Medical Sciences,

Karwar, Karnataka, India

Santosh S Jeevannavar

Professor, SDM College of

Medical Sciences, Dharwad,

Karnataka, India
Corresponding Author: Shreesha Tokur

Assistant Professor, Karwar Institute of Medical Sciences, Karwar, Karnataka, India

\section{Analysis of the short term functional results of osteochondral defects in a stable knee treated by arthroscopic debridement and micro fracture technique at 1 year follow up using the lysholm knee scoring scale}

\section{Shreesha Tokur and Santosh S Jeevannavar}

DOI: https://doi.org/10.22271/ortho.2020.v6.i4d.2348

\begin{abstract}
Introduction: Articular cartilage is a complex tissue with an ability to withstand tremendous forces over many cycles and years, but at the same time it has no ability to heal even after a minor injury. The incidence of articular cartilage injuries varies from $60-66 \%$ during routine arthroscopic surgery ${ }^{[1,2]}$.

Objectives: To analyze the short term functional results of Osteochondral defects in a stable knee treated by Arthroscopic Debridement and Micro fracture technique at 1 year follow up using the Lysholm Knee Scoring Scale.

Methodology: Source of data: All the patients with osteochondral injuries who were managed by arthroscopic micro fracture technique, and have come for follow up. All the patients who underwent arthroscopy for suspected intra-articular pathology and were diagnosed intraoperatively with osteochondral defects, and managed by micro fracture. All the patients admitted to SDM Hospital that are scheduled to undergo arthroscopic debridement and micro fracture for osteochondral defects of the knee based on clinical and MRI findings, who fit into inclusion criteria, within a period of 1 year, from November 2012 to October 2013.

Conclusion: Articular cartilage injuries are more frequently encountered during routine arthroscopic surgeries of the knee, than thought. Arthroscopic debridement and micro fracture results in an improved functional outcome at 1 year follow up. Marrow stimulation by micro fracture technique does not require any specialized instruments or operative setup, and can be performed in any center where routine arthroscopic surgeries of the knee are done. Arthroscopic debridement and micro fracture is a single staged procedure as compared to Autologous Chondrocyte Implantation. The cost involved in arthroscopic debridement and micro fracture is far less compared to Autologous Chondrocyte Implantation and other cell based procedures. In comparison to other management modalities for osteochondral defects like Mosaicplasty, Autologous Chondrocyte Implantation and other cell based techniques, Micro fracture does not come in the way for repeat surgeries if required. We safely recommend Arthroscopic debridement and Micro fracture technique as a 'first line' management for the treatment of osteochondral defects in a stable knee, for lesions less than $4 \mathrm{~cm}^{2}$ in size.
\end{abstract}

Keywords: Osteochondral defects, arthroscopic debridement, micro fracture technique, lysholm knee scoring scale

\section{Introduction}

Articular cartilage is a complex tissue with an ability to withstand tremendous forces over many cycles and years, but at the same time it has no ability to heal even after a minor injury. The incidence of articular cartilage injuries varies from $60-66 \%$ during routine arthroscopic surgery ${ }^{[1,2]}$.

Many of these particular injuries occur in the young and have been identified as an important cause of permanent disability because of the high mechanical joint stress. Articular cartilage injuries are difficult to diagnose on clinical examination alone and also on routine radiographs [3]. On MRI, special sequences are required to outline cartilage injuries.

Several treatment options have been advocated in the treatment of Osteochondral defects of the Knee, ranging from Arthroscopic Debridement and Micro fracture, Abrasion Chondroplasty, 
Mosaicplasty to Autologous Chondrocyte Implantation. Apart from Arthroscopic Debridement and Micro fracture, the treatment cost of other surgical modalities is expensive.

Arthroscopic debridement and micro fracture was first described by Steadman about 20 years ago with good results and is recommended as a treatment modality for moderate sized defects with minimal malalignment. Micro fracture is a technically simple and cost-effective treatment option for articular cartilage lesions of the knee. It does not prevent the later use of other, more complex procedures. It also has the added advantage of low risk of patient morbidity and hence reducing the hospital stay of the patient. ${ }^{[4,5]}$

\section{Objectives}

To analyze the short term functional results of Osteochondral defects in a stable knee treated by Arthroscopic Debridement and Micro fracture technique at 1 year follow up using the Lysholm Knee Scoring Scale.

\section{Methodology}

Source of data: All the patients with osteochondral injuries who were managed by arthroscopic micro fracture technique, and have come for follow up. All the patients who underwent arthroscopy for suspected intra-articular pathology and were diagnosed intraoperatively with osteochondral defects, and managed by micro fracture. All the patients admitted to SDM Hospital that are scheduled to undergo arthroscopic debridement and micro fracture for osteochondral defects of the knee based on clinical and MRI findings, who fit into inclusion criteria, within a period of 1 year, from November 2012 to October 2013.

\section{Type of Study}

Combined Retrospective and Prospective study design.

\section{Method of collection}

The sampling method to be applied for collection of cases will be convenient sampling.

1. Source of Data

2. Clinical examination to be done by 2 Orthopedic Surgeons and arthroscopic examination done by the other Orthopedic Surgeon.

3. MRI report collected from Radio Diagnosis Department.

\section{Inclusion Criteria}

All patients admitted to SDM Hospital, aged between 18 and 50 years who are diagnosed with Osteochondral defect in a stable knee, with the aid of MRI Scan, and are treated by arthroscopic debridement and micro fracture technique.

\section{Exclusion Criteria}

1. Patients diagnosed with ACL and PCL injuries in association with osteochondral defect, who underwent additional reconstruction procedures.

2. Presence of fractures in and around the knee joint.

3. Varus and valgus malalignment of the knee with osteoarthritis, as determined by X-Ray of the knee joints.

4. Age below 18 and above 50 years.

5. Other inflammatory arthritic conditions of the knee.

\section{Study Area}

The study will be conducted in the SDM College of Medical Sciences and Hospital, Dharwad.

\section{Results}

In our study, a total of 50 cases were included with their ages ranging from $23-50$ years. The patients were divided into 2 age groups, with the maximum number of cases seen between $36-50$ years.

Table 1: Age wise distribution of all patients

\begin{tabular}{|c|c|c|}
\hline Age Category (in years) & Frequency & Percentage (in \%) \\
\hline $18-35$ & 21 & 42 \\
\hline $36-50$ & 29 & 58 \\
\hline Total & 50 & 100 \\
\hline
\end{tabular}

Table 2: Mode of Injury in the study group

\begin{tabular}{|c|c|c|}
\hline Type of injury & Frequency & Percentage (in \%) \\
\hline No Trauma & 23 & 46 \\
\hline Road Traffic Accident (RTA) & 05 & 10 \\
\hline Twisting injury & 16 & 32 \\
\hline Fall from height & 06 & 12 \\
\hline Total & 50 & 100 \\
\hline
\end{tabular}

Table 3: Site of osteochondral lesion on arthroscopy

\begin{tabular}{|c|c|c|}
\hline Site of Lesion & Frequency & $\boldsymbol{\%}$ \\
\hline Medial Femoral Condyle (MFC) & 39 & 78 \\
\hline Lateral Femoral Condyle (LFC) & 07 & 14 \\
\hline Medial Femoral and Tibial Condyle (MFC +MTC) & 02 & 04 \\
\hline Lateral Tibial Condyle (LTC) & 02 & 04 \\
\hline Total & 50 & 100 \\
\hline \multicolumn{2}{|c}{}
\end{tabular}

Table 4: Correlation of the body mass index (BMI) with the knee function score after 1 year following arthroscopic micro fracture

\begin{tabular}{|c|c|c|c|c|c|}
\hline \multirow{2}{*}{ B.M.I } & \multicolumn{4}{|c|}{ Lysholm Score at 1 year } & \multirow{2}{*}{ Total } \\
\cline { 2 - 5 } & Excellent & Good & Fair & Poor & \\
\hline Normal & 01 & 11 & 03 & 0 & 15 \\
\hline Overweight & 0 & 11 & 17 & 01 & 29 \\
\hline Obesity & 0 & 0 & 03 & 03 & 06 \\
\hline Total & 01 & 22 & 23 & 04 & 50 \\
\hline
\end{tabular}

The correlation of the Body Mass Index with Lysholm Knee Score was performed

using the Pearson Co-efficient test, and the co-efficient of variation ( $p$ value) was calculated. ' $p$ ' value of $<0.05$ was considered significant.

\begin{tabular}{|c|c|c|c|}
\hline Test & Value & Degree of freedom & P Value \\
\hline Pearson Chi Square & 26.560 & 6 & 0.000 \\
\hline Likelihood Ratio & 23.439 & 6 & 0.000 \\
\hline Number of Cases & 50 & & \\
\hline
\end{tabular}

The correlation of BMI with Functional outcome of micro fracture at 1 year follow up is statistically significant.

Table 5: Arthroscopic Lesion Size of all the patients in the study group

\begin{tabular}{|c|c|c|}
\hline Lesion Size (in $\mathbf{~ c m}^{2}$ ) & Frequency & Percentage (in \%) \\
\hline Upto 2 & 26 & 52 \\
\hline $2.1-3$ & 21 & 42 \\
\hline 3.1 and more & 03 & 06 \\
\hline Total & 50 & 100 \\
\hline
\end{tabular}

In the study group of 50 patients, 26 patients had a chondral lesion size of $2 \mathrm{~cm}^{2}$ or less, followed by 21 patients with lesion size ranging from $2.1-3 \mathrm{~cm}^{2}$.

Only 3 patients in our study group presented with chondral lesion size of more than $3 \mathrm{~cm}^{2}$. 
Table 6: Correlation between osteochondral lesion size with knee function after 1 year following arthroscopic micro fracture

\begin{tabular}{|c|c|c|c|}
\hline \begin{tabular}{|c|} 
Lesion Size \\
(in $\left.\mathbf{c m}^{2}\right)$
\end{tabular} & $\begin{array}{l}\text { Mean Pre-OP } \\
\text { Lysholm Score }\end{array}$ & $\begin{array}{l}\text { Mean Postop } \\
\text { Lysholm Score }\end{array}$ & $\begin{array}{l}\text { Standard } \\
\text { Deviation }\end{array}$ \\
\hline Upto 2 & 66.96 & 81.96 & 7.754 \\
\hline $2.1-3.0$ & 63.95 & 80.95 & 7.386 \\
\hline 3.1 and More & 62.0 & 68.0 & 16.823 \\
\hline
\end{tabular}

The correlation between the arthroscopic lesion size with knee function at 1 year follow up was performed using ANOVA test, to detect statistical significance between the 3 groups. 'p' value $<0.05$ was considered significant.

The results demonstrate a statistical significance between the lesion size and post-operative functional outcome at 1 year follow up. The correlation between the 3 groups of lesion size and improvement in the Lysholm Score is also statistically significant, with patients who had a lesion size of less than 2 $\mathrm{cm}^{2}$ demonstrating a better functional outcome.

\section{Discussion}

The diagnosis of osteochondral injuries of the knee is difficult, due to the fact that majority of the cases are clinically misdiagnosed as either meniscal or ligamentous injuries. A review of 31,516 knee arthroscopies by Curl WW et al $^{[1]}$, revealed the presence of chondral injuries in $63 \%$ cases, of which $68 \%$ had an associated meniscal pathology. Hjelle et al ${ }^{[6]}$, conducted a similar review of 1,000 arthroscopies, and demonstrated chondral lesions in $61 \%$ cases, of which $42 \%$ were associated with meniscal injury.

In our study involving a total of 50 patients, meniscal pathology was seen in $66 \%$ of the cases, which is comparable to other studies. This can be explained by the fact that, majority of the patients were initially evaluated for a meniscal or a ligamentous pathology, and were diagnosed with osteochondral defects during arthroscopic examination.

The inherent challenge of accurate clinical diagnosis of osteochondral injuries of the knee, has led most orthopaedic surgeons to rely on Magnetic Resonance Imaging and Arthroscopy for its diagnosis and management. The imaging modality employed in our study is also MRI. All the patients in our study underwent MRI evaluation of the affected knee joint with 'cartilage' specific sequences, in a 1.5 Tesla machine. $34 \%$ of the patients in our study were diagnosed with Osteochondral injuries intraoperatively. This low accuracy rate in detecting an osteochondral defect through MRI can be explained by the fact that majority of the patients in our study, underwent MRI imaging for a clinically diagnosed meniscal pathology.

The treatment of articular cartilage injuries has evolved from the use of osteoarticular implants in the past, to the chondral reparative and restorative procedures of the present. The current chondral resurfacing techniques have evolved from Pridie's subchondral bone drilling ${ }^{7}$ procedure, which required arthrotomy of the knee. This resulted in many patients treated by Pridie's technique to develop post-operative knee stiffness. The advent of arthroscopy has augmented the refinement of these bone marrow stimulation procedures.

The arthroscopic micro fracture technique, developed by Dr. J.R. Steadman ${ }^{[8]}$, is a marrow stimulation procedure, based on the principle of stimulation of the subchondral bone by micro fracture, leading to activation of the intrinsic repair mechanism, by the release of pluripotent mesenchymal cells. This leads to the formation of a fibrocartilage cover filling the chondral defect. Other articular cartilage repair strategies include Autologous Chondrocyte Implantation, Mosaicplasty, and Osteochondral Autologous Transfer, which have been shown to produce a histo-morphologically superior reparative cartilage. However, these procedures are expensive, technically demanding, multi staged, and require specialized medical infrastructure for its implementation.

The clinical outcome of the patients in our study was assessed by the use of Lysholm-Gillquist Knee Scoring Scale, pre-operatively and post operatively at 1 month, 6 months and 1 year follow up. The results of the functional outcome in our study are comparable to other studies.

Symptomatic assessment of the patients in our study was also done through the Lysholm Knee Scoring scale, and the results assessing Pain and Knee swelling were comparable with other studies.

In our study of 50 patients treated with arthroscopic micro fracture, we assessed the symptomatic parameters of the patients, including pain and swelling of the knee joint, through the Lysholm Knee Scoring Scale, and compared the preoperative and postoperative results.

37 patients $(74 \%)$ demonstrated an excellent improvement in pain at the knee joint, 1year post-surgery, which was compared with pain score preoperatively. The results demonstrated that the improvement in the knee pain was statistically significant ( $p$ value $<0.03$ ). The result of our study is comparable to the studies of Steadman et al, Miller et al, Gobbi et al, Mithoefer et al, and Kon et al ${ }^{[9]}$.

34 patients $(68 \%)$ demonstrated an excellent improvement in knee swelling, at 1 year follow up. 13 patients demonstrated a persistence of the knee swelling at 6 months postoperative follow up, which had improved at 1-year post-operative follow up. Only 3 patients in our study had persistence of pain and swelling of the knee at 1 year follow up, and were advised a second MRI scan of the knee joint, to assess the status of healing of the osteochondral defect.

We also documented the pre injury activity levels of all the patients in our study, and analyzed the number of patients who returned to their pre injury activity levels following micro fracture. The results were compared to other studies which assessed a similar parameter.

The results of our study has demonstrated a strong statistical significance in correlating the Body Mass Index, return to pre injury activity levels, and the arthroscopic size of osteochondral defect, to the overall functional outcome at 1 year follow up, as assessed by the Lysholm Knee Scoring Scale. This is in strong agreement with the studies of Steadman et al, Miller et al, Asik et al, who noticed a similar correlation in their studies.

A relook arthroscopy was performed in 2 patients, 1 -year post surgery, to assess the fill and quality of the reparative cartilage. The reparative cartilage had completely filled the micro fracture area, and was found to have a similar consistency with the surrounding intact articular cartilage.

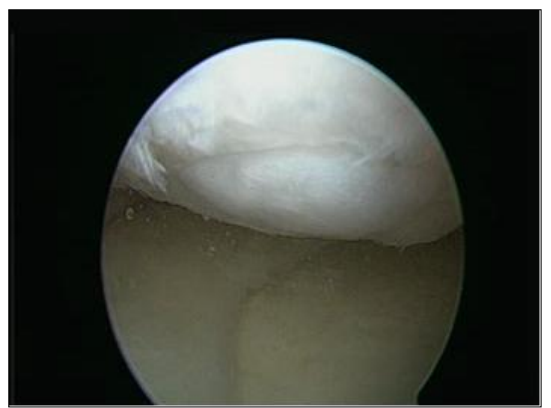

Fig. 1: Relook Arthroscopy: Reparative cartilage filling the chondral defect 


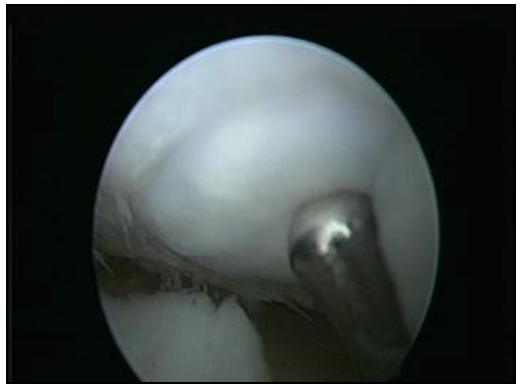

Fig 2: Assessment of reparative cartilage quality by Probing

All the patients in the study group were advised to undergo an MRI scan 1 year following the microfracture procedure, to assess the progression of healing of the osteochondral defects and also the quality of reparative cartilage. However, due to financial constraints of the patients, the MRI scan was performed in 3 patients who had persistent knee swelling and consented for a second scan, which showed good regenerate of cartilage.

\section{Conclusion}

- Articular cartilage injuries are more frequently encountered during routine arthroscopic surgeries of the knee, than thought.

- Arthroscopic debridement and microfracture results in an improved functional outcome at 1 year follow up.

- Marrow stimulation by microfracture technique does not require any specialized instruments or operative setup, and can be performed in any centre where routine arthroscopic surgeries of the knee are done.

- Arthroscopic debridement and microfracture is a single staged procedure as compared to Autologous Chondrocyte Implantation.

- The cost involved in arthroscopic debridement and microfracture is far less compared to Autologous Chondrocyte Implantation and other cell based procedures.

- In comparison to other management modalities for osteochondral defects like Mosaicplasty, Autologous Chondrocyte Implantation and other cell based techniques, Microfracture does not come in the way for repeat surgeries if required.

- We safely recommend Arthroscopic debridement and Microfracture technique as a 'first line' management for the treatment of osteochondral defects in a stable knee, for lesions less than $4 \mathrm{~cm}^{2}$ in size.

\section{References}

1. Curl WW, Krome J, Gordon ES. Cartilage injuries: a review of 31,516 knee arthroscopies. Arthroscopy. 1997; 13:456.

2. Aroen A, Loken S, Heir S, Alvik E, Ekeland A, Granlund OG et al. Articular cartilage lesions in 993 consecutive knee arthroscopies. Am J Sports Med. 2004; 32:211-5.

3. Erggelet C, Mandelbaum BR. Principles of cartilage repair. Germany: Steinkopff Verlag, 2008.

4. Steadman JR, Rodkey WG, Singleton SB, Briggs KK. Microfracture technique for full-thickness chondral defects: Technique and clinical results. Oper Tech Orthop. 1997; 7:300-304.

5. Feagin JA, Steadman JR, editors. Crucial Principles in Care of the Knee. Lippincott Williams \& Wilkins, 2008.

6. Lysholm J, Gillquist J. Evaluation of knee ligament surgery results with special emphasis on use of a scoring scale. Am J Sports Med. 1982; 10(3):150-154.

7. Pridie KH. A method of resurfacing osteoarthritic knee joints. In: Proceedings of the British Orthopaedic Association. J Bone Joint Surg Br. 1959; 41:618-9.

8. Steadman JR, Rodkey WG, Rodrigo JJ. Microfracture: Surgical Technique and Rehabilitation to Treat Chondral Defects. Clin Orthop Relat Res. 2001; 391:S362-369.

9. Kon E, Filardo G, Berruto M, Benazzo F, Zanon G, Della Villa $S$ et al. Articular cartilage treatment in high-level male soccer players: a prospective comparative study of arthroscopic second-generation autologous chondrocyte implantation versus microfracture. Am J Sports Med. 2011; 39(12):2549-57. 